Marquette University

e-Publications@Marquette

Electrical and Computer Engineering Faculty

Electrical and Computer Engineering, Department

Research and Publications

$1-1-2014$

\title{
Robust and Resilient State-dependent Control of Continuous-time Nonlinear Systems with General Performance Criteria
}

\author{
Xin Wang \\ Southern Illinois University Edwardsville \\ Edwin E. Yaz \\ Marquette University, edwin.yaz@marquette.edu \\ James Long \\ Oregon Institute of Technology
}

Published version. Systems Science \& Control Engineering, Vol. 2, No. 1 (2014): 34-40. DOI. (C) 2014

The Authors. Used with permission.

This is an open-access article distributed under the terms of the Creative Commons Attribution License. 


\title{
Robust and resilient state-dependent control of continuous-time nonlinear systems with general performance criteria
}

\author{
Xin Wang ${ }^{\mathrm{a} *}$, Edwin E. Yaz ${ }^{\mathrm{b}}$ and James Long ${ }^{\mathrm{c}}$ \\ ${ }^{a}$ Department of Electrical and Computer Energy Engineering, Southern Illinois University Edwardsville, Edwardsville, IL 62026, USA; \\ ${ }^{b}$ Department of Electrical and Computer Engineering, Marquette University, Milwaukee, WI 53201, USA; ${ }^{c}$ Computer Systems \\ Engineering, Oregon Institute of Technology, Klamath Falls, OR 97601, USA
}

(Received 19 November 2013; final version received 18 December 2013)

\begin{abstract}
A novel state-dependent control approach for continuous-time nonlinear systems with general performance criteria is presented in this paper. This controller is optimally robust for model uncertainties and resilient against control feedback gain perturbations in achieving general performance criteria to secure quadratic optimality with inherent asymptotic stability property together with quadratic dissipative type of disturbance reduction. For the system model, unstructured uncertainty description is assumed, which incorporates commonly used types of uncertainties, such as norm-bounded and positive real uncertainties as special cases. By solving a state-dependent linear matrix inequality at each time, sufficient condition for the control solution can be found which satisfies the general performance criteria. The results of this paper unify existing results on nonlinear quadratic regulator, $H_{\infty}$ and positive real control. The efficacy of the proposed technique is demonstrated by numerical simulations of the nonlinear control of the inverted pendulum on a cart system.
\end{abstract}

Keywords: robust control; nonlinear systems; optimal control

\section{Introduction}

Optimal control of nonlinear systems is traditionally characterized in terms of Hamilton Jacobi Equations (HJEs). The solution of the HJEs provides the necessary and sufficient optimal control condition for nonlinear systems. Furthermore, when the controlled system is linear timeinvariant and the performance index is linear quadratic regulator (LQR), the HJEs reduced to Algebraic Riccati Equations (AREs). As for $H_{\infty}$ nonlinear control problem, the optimal control solution is equivalent to solving the corresponding Hamilton Jacobi Inequalities (HJIs) Basar and Bernhard (1995). However, HJEs and HJIs, which are firstorder partial differential equations and inequalities, cannot be solved for more than a few state variables. In the past few years, it has been shown that the problems of quadratic regulation and $H_{\infty}$ nonlinear control can be approached by the state-dependent Riccati equation (SDRE) and nonlinear matrix inequality (NLMI) techniques (Cloutier, 1997; Cloutier, D'Souza, \& Mracek, 1996; Huang \& Lu, 1996). The state-dependent linear matrix inequality (LMI) control of nonlinear systems, as pointed out in Wang and Yaz (2009), Wang, Yaz, and Jeong (2010), and Wang, Yaz, and Yaz $(2010,2011)$ synthesizes a controller to achieve mixed nonlinear quadratic regulator (NLQR) and $H_{\infty}$ control objectives.
Dissipative control for linear systems has also received considerable attention over the past two decades. The concept of dissipative systems was first introduced by Willems (1972a, 1972b), and further generalized by Hill and Moylan $(1975,1976,1980)$, playing an important role in systems, circuits and controls. The theory of dissipative systems generalizes the basic tools including the passivity theorem, bounded real lemma, Kalman-Yakubovich lemma and circle criterion. Dissipativity performance includes $H_{\infty}$ performance, passivity, positive realness and sector-bounded constraint as special cases. Research addressing the problems of $H_{\infty}$ and positive real control systems can be found in Zhou and Khargonekar (1988) Doyle, Glover, Khargonekar, and Francis (1989), Haddad and Bernstein (1991), Sun, Khargonekar, and Shim (1994), Safonov, Jonckheere, Verma, and Limebeer (1987) and Shim (1996). Control of uncertain linear systems with $L_{2}$-bounded structured uncertainty satisfying $H_{\infty}$ and passivity criteria has been tackled in Khargonekar, Petersen, and Zhou (1990) and Petersen (1987). More recent development involving the quadratic dissipative control for linear systems problem has been tackled in Tan, Soh, and Xie (2000) and Xie, Xie, and De Souza (1998).

In this paper, we further consider the problem of optimal, robust and resilient LMI control of continuous-time

\footnotetext{
*Corresponding author. Email: xwang@siue.edu
} 
nonlinear systems with general performance criteria. The controller is robust for model uncertainties and resilient for control gain perturbations. As for uncertain nonlinear systems, we consider a general form of $L_{2}$-bounded uncertainty description, without any standard structure, incorporating commonly used types of uncertainty, such as norm-bounded and positive real uncertainties as special cases. The purpose behind this novel approach is to convert a nonlinear system control problem into a convex optimization problem which is solved by state-dependent LMI. The recent development in convex optimization provides very efficient means for solving LMIs. If a solution can be expressed in a LMI form, then there exist optimization algorithms providing efficient global numerical solutions (Boyd, Ghaoui, Feron, \& Balakrishnan, 1994). Therefore, if the LMI is feasible, the LMI control technique provides asymptotically stable solutions satisfying various general performance criteria. We further propose to employ general performance criteria to design the controller guaranteeing the quadratic suboptimality with inherent stability property in combination with dissipativity type of disturbance attenuation. The general performance criteria are a generalization of the NLQR, $H_{\infty}$, positive realness and sector-bounded constraint; therefore, the results of the paper unify existing control results and provide a more general control design framework.

The paper is organized as follows. In Section 2, we present the general performance criteria including the performance of NLQR, $H_{\infty}$, positive realness and sectorbounded constraint. Section 3 presents the state-dependent LMI-based control for nonlinear systems achieving general performance criteria. Finally, the inverted pendulum on a cart is used for applying the algorithm to an under-actuated robot with nonlinear dynamics to examine the effectiveness and robustness of the new approach in Section 4.

\section{System model and general performance criteria analysis}

The following notation is used in this work: $\mathfrak{R}_{+}$stands for the set of non-negative real numbers and $\Re^{n}$ stands for the $n$-dimensional Euclidean space. $x \in \mathfrak{R}^{n}$ denotes $n$-dimensional real vector with norm $\|x\|=\left(x^{\mathrm{T}} x\right)^{1 / 2}$, where $(\cdot)^{\mathrm{T}}$ indicates transpose. $\Re^{n \times m}$ is the set of $n \times m$ real matrices. $I_{n}$ is the $n \times n$ identity matrix. $A \geq 0$ for a symmetric matrix denotes a positive semi-definite matrix. $L_{2}$ is the space of finite dimensional vectors with finite energy: $\int_{0}^{\infty}|x(t)|^{2} \mathrm{~d} t<\infty$. Let $L_{2 e}^{n}$ be the extended space of $L_{2}$ space defined by

$$
\begin{aligned}
L_{2 e}^{n}= & \left\{f: f \text { is a measureable function }: \Re_{+} \rightarrow \mathfrak{R}^{n},\right. \text { with } \\
& \left.\times \text { property that } F_{T} f \in L_{2} \text { for all finite } T \in \Re_{+}\right\}
\end{aligned}
$$

where $F_{T} f(t)=\left\{\begin{array}{ll}f(t), & 0 \leq t \leq T \\ 0, & T<t\end{array}\right.$ is called the truncation function on $\Re_{+}$with values in $\Re^{n}$. The inner product in this space is defined as $\langle u(t), v(t)\rangle_{T}=\int_{0}^{T} u(t) v(t) \mathrm{d} t$, for $u$, $v \in L_{2 e}^{n}$.

Consider the following nonlinear dynamical system equation and performance output equation

$$
\begin{aligned}
\dot{x}= & f(x(t), u(t), w(t)), \\
= & \left(A(x, t)+\Delta_{A}(x, t)\right) x(t)+\left(B(x, t)+\Delta_{B}(x, t)\right) u(t) \\
& +\left(E(x, t)+\Delta_{E}(x, t)\right) w(t), \\
= & \left(A+\Delta_{A}\right) x+\left(B+\Delta_{B}\right) u+\left(E+\Delta_{E}\right) w \\
z(t)= & g(x(t), w(t))=C \cdot x+D \cdot w,
\end{aligned}
$$

where $x(t) \in \Re^{n}$ is the state variable of the dynamical system, $u(t) \in \Re^{m}$ the applied input, $w(t) \in \Re^{p}$ the $L_{2}$ type of disturbance, $z(t) \in \mathfrak{R}^{r}$ the performance output function, $f, g$ the smooth real vector functions, $A \in \Re^{n \times n}, B \in \Re^{n \times m}, E \in$ $\Re^{n \times p}, C \in \mathfrak{R}^{r \times n}$ and $D \in \mathfrak{R}^{r \times p}$ the state-dependent coefficient matrices, and $\Delta_{A} \in \Re^{n \times n}, \Delta_{B} \in \Re^{n \times m}$ and $\Delta_{E} \in \Re^{n \times p}$ the time-varying uncertainty matrices.

It is assumed that the state feedback is available and the state feedback control input is given by

$$
u(t)=\left(K(x, t)+\Delta_{K}(x, t)\right) x(t)=\left(K+\Delta_{K}\right) x .
$$

Introducing the following quadratic energy supply function $E: L_{2 e}^{r} \times L_{2 e}^{p} \times \Re^{+} \rightarrow \Re$ associated with the system equations, defined in Hill and Moylan (1975, 1976, 1980):

$$
E(z, w, T)=\langle z, Q z\rangle_{\mathrm{T}}+2\langle z, S w\rangle_{\mathrm{T}}+\langle w, R w\rangle_{\mathrm{T}},
$$

where $Q \in \mathfrak{R}^{r \times r}, S \in \mathfrak{R}^{r \times p}, R \in \mathfrak{R}^{p \times p}$ are the chosen weighing matrices. Next, from the definition of dissipativity (Hill \& Moylan 1975, 1976, 1980), we have

Definition 1 Given matrices $Q \in \mathfrak{R}^{r \times r}, S \in \mathfrak{R}^{r \times p}, R \in$ $\Re^{p \times p}$ with $Q, R$ symmetric, the system (1) and (2) with energy function (4) is said to be $(Q, S, R)$ dissipative if for some real function $\beta(\cdot)$ with $\beta(0)=0$. The physical meaning for $\beta(\cdot)$ is the stored energy at the initial time, given by the initial condition $x_{0}$.

$$
E(z, w, T)+\beta\left(x_{0}\right) \geq 0, \forall w \in L_{2 e}, \forall T \geq 0 .
$$

Furthermore, iffor some scalar $\alpha>0$,

$$
E(z, w, T)+\beta\left(x_{0}\right) \geq \alpha\langle w, w\rangle_{\mathrm{T}}, \forall w \in L_{2 e}, \forall T \geq 0 .
$$

The system (1) and (2) is said to be strictly $(Q, S, R)$ dissipative.

THEOREM 1 Consider the quadratic function $V=x^{\mathrm{T}} P x>$ 0, matrices $Q \in \Re^{r \times r}, S \in \Re^{r \times p}, R \in \Re^{p \times p}$ with $Q, R$ symmetric, $M \in \Re^{n \times n}, M>0, N \in \mathfrak{R}^{m \times m}, N>0$ with $M, N$ symmetric, the control of nonlinear system (1) and (2) will achieve mixed NLQR and dissipative performance if the following condition holds:

$$
\begin{aligned}
\dot{V} & +x^{\mathrm{T}} M x+u^{\mathrm{T}} N u \\
& -\left(z^{\mathrm{T}} Q z+2 z^{\mathrm{T}} S w+w^{\mathrm{T}} R w\right)<0, \forall T \geq 0 .
\end{aligned}
$$


Proof By integrating Equation (7) from 0 to $T$, we have

$$
\begin{aligned}
& \int_{0}^{T}\left(z^{\mathrm{T}} Q z+2 z^{\mathrm{T}} S w+w^{\mathrm{T}} R w\right) \mathrm{d} t> \\
& \int_{0}^{T} x^{\mathrm{T}} M x \mathrm{~d} t+\int_{0}^{T} u^{\mathrm{T}} N u \mathrm{~d} t+V(x(T)) \\
& \quad-V(x(0)), \forall T \geq 0 .
\end{aligned}
$$

Let $\beta\left(x_{0}\right)=V(x(0)), V(x)=x^{\mathrm{T}} P x, V(x(T)) \geq 0$, Equation (8) implies

$$
\int_{0}^{T}\left(z^{\mathrm{T}} Q z+2 z^{\mathrm{T}} S w+w^{\mathrm{T}} R w\right) \mathrm{d} t+\beta\left(x_{0}\right)>0, \forall T \geq 0,
$$

which is the condition of $(Q, S, R)$ dissipative. By adding the terms $x^{\mathrm{T}} M x+u^{\mathrm{T}} N u$, we include the NLQR control performance into the original $(Q, S, R)$-dissipative criteria.

Remark 1 Notice that both $H_{\infty}$ and passivity are special cases of $(Q, S, R)$ dissipativity. The special cases are summarized as follows:

Case $1 Q=-I, S=0, R=\gamma^{2} I$, the $\operatorname{strict}(Q, S, R)$ dissipativity reduces to $H_{\infty}$ design (Doyle et al., 1989). The overall control design satisfies mixed $\mathrm{NLQR}-H_{\infty}$ performance.

Case $2 Q=0, S=I, R=0$, the strict $(Q, S, R)$ dissipativity reduces to strict positive realness (Sun et al., 1994). The overall control design satisfies mixed NLQR-strict positive realness performance.

Case $3 Q=-\theta I, S=(1-\theta) I, R=\theta \gamma^{2} I$, the strict $(Q$, $S, R)$ dissipativity reduces to mixed $H_{\infty}$ and positive real performance design, when $\theta \in(0,1)$. The overall control design satisfies mixed NLQR $-H_{\infty}-$ positive real performance.

Case $4 Q=-I, S=\frac{1}{2}\left(K_{1}+K_{2}\right)^{\mathrm{T}}, R=-\frac{1}{2}\left(K_{1}^{\mathrm{T}} K_{2}+K_{2}^{\mathrm{T}}\right.$ $K_{1}$ ), where $K_{1}$ and $K_{2}$ are constant matrices of appropriate dimensions, the strict $(Q, S, R)$ dissipativity reduces to a sector-bounded constraint (Gupta \& Joshi, 1994). The overall control design satisfies mixed NLQR-sector-bounded constraint performance.

Before introducing the main result of the paper, the following model of unstructured uncertainties is introduced.

AsSUMPTION 1 The following general form of $L_{2}$-bounded unstructured uncertainties is considered:

$$
\begin{aligned}
\Delta_{A} \Delta_{A}^{\mathrm{T}} & \leq \gamma_{A} I, \\
\Delta_{B} \Delta_{B}^{\mathrm{T}} & \leq \gamma_{B} I, \\
\Delta_{E} \Delta_{E}^{\mathrm{T}} & \leq \gamma_{E} I, \\
\Delta_{K} \Delta_{K}^{\mathrm{T}} & \leq \gamma_{K} I,
\end{aligned}
$$

for $\forall x \in \Re^{n}$ and $t \geq 0$.

\section{State-dependent LMI control}

LEMMA 1

$$
A B^{\mathrm{T}}+B A^{\mathrm{T}} \leq \alpha A A^{\mathrm{T}}+\alpha^{-1} B B^{\mathrm{T}} .
$$

This can be proven easily by considering

$$
\left(\alpha^{1 / 2} A-\alpha^{-1 / 2} B\right)\left(\alpha^{1 / 2} A-\alpha^{-1 / 2} B\right)^{\mathrm{T}} \geq 0 .
$$

Also, by choosing $A$ and $B$ matrices as $A=\left[\begin{array}{c}a^{\mathrm{T}} \\ 0\end{array}\right]$ and $B=$ $\left[\begin{array}{c}0 \\ b^{\mathrm{T}}\end{array}\right]$, we have

$$
\left[\begin{array}{cc}
0 & a^{\mathrm{T}} b \\
b^{\mathrm{T}} a & 0
\end{array}\right] \leq\left[\begin{array}{cc}
\zeta a^{\mathrm{T}} a & 0 \\
0 & \zeta^{-1} b^{\mathrm{T}} b
\end{array}\right]
$$

Lemma 2 Denote $X=P^{-1}$ for positive definite matrix $P>0$, then the following equality always holds:

$$
X \dot{P} X=-\dot{X} \text {. }
$$

This can be proven easily by considering

$$
0=\frac{\mathrm{d}}{\mathrm{d} t}(I)=\frac{\mathrm{d}}{\mathrm{d} t}\left(P P^{-1}\right)=\frac{\mathrm{d}}{\mathrm{d} t}(P) \cdot P^{-1}+P \cdot \frac{\mathrm{d}}{\mathrm{d} t}\left(P^{-1}\right) .
$$

Remark 2 Since in the presentation below, $P$ will be used to describe the energy content, which needs to decrease due to the asymptotic stability requirement, $X=P^{-1}$ matrix will be increasing in time. Therefore, we have $X \dot{P} X=$ $-\dot{X}<0$.

The following theorem summarizes the main results of the paper:

THEOREM 2 Given the system Equation (1), performance output Equation (2) and control Equation (3), if there exist matrices $X=P^{-1}>0$ and $Y$ for all $t>0$, such that the following state-dependent LMI holds:

$$
\left[\begin{array}{cccc}
\Upsilon_{11} & \Upsilon_{12} & X & Y^{\mathrm{T}} \\
* & \Upsilon_{22} & 0 & 0 \\
* & * & \Upsilon_{33} & 0 \\
* & * & * & \Upsilon_{44}
\end{array}\right]<0
$$

where

$$
\begin{aligned}
\Upsilon_{11}= & X A^{\mathrm{T}}+A X+Y^{\mathrm{T}} B^{\mathrm{T}}+B Y+B B^{\mathrm{T}} \\
& +\left[\gamma_{A}+2 \gamma_{B}+\gamma_{E}\right] I, \\
\Upsilon_{12}= & E-X C^{\mathrm{T}} Q D-X C^{\mathrm{T}} S, \\
\Upsilon_{22}= & -D^{\mathrm{T}} Q D-2 D^{\mathrm{T}} S-R+I, \\
\Upsilon_{33}= & -\left\{I+\left[3+\lambda_{\max }(N)\right] \gamma_{K} I+M+C^{\mathrm{T}} Q C\right\}^{-1}, \\
\Upsilon_{44}= & -\left\{I+N^{2}+N\right\}^{-1} .
\end{aligned}
$$

Then the inequality (7), which guarantees mixed NLQR and dissipative performance, is satisfied. The nonlinear feedback control gain is given by

$$
K=Y \cdot P \text {. }
$$


Proof In the proof below, the time and state argument will be dropped for notational simplicity. By applying system and performance output Equations (1) and (2), and state feedback input Equation (3), the performance index can be formed as follows:

$$
\begin{aligned}
x^{\mathrm{T}} & \left\{A+\Delta_{A}+\left(B+\Delta_{B}\right)\left(K+\Delta_{K}\right)\right\}^{\mathrm{T}} P x+w^{\mathrm{T}}\left[E+\Delta_{E}\right]^{\mathrm{T}} P x \\
& +x^{\mathrm{T}} P\left\{A+\Delta_{A}+\left(B+\Delta_{B}\right)\left(K+\Delta_{K}\right)\right\} x \\
& +x^{\mathrm{T}} P\left[E+\Delta_{E}\right] w \\
& +x^{\mathrm{T}} \dot{P} x+x^{\mathrm{T}} M x+x^{\mathrm{T}}\left[K+\Delta_{K}\right]^{\mathrm{T}} N\left[K+\Delta_{K}\right] x \\
& -[C x+D w]^{\mathrm{T}} Q[C x+D w]-2[C x+D w]^{\mathrm{T}} S w \\
& -w^{\mathrm{T}} R w<0 .
\end{aligned}
$$

Equivalently,

$$
\left[\begin{array}{ll}
x^{\mathrm{T}} & w^{\mathrm{T}}
\end{array}\right] \Psi\left[\begin{array}{ll}
x & w
\end{array}\right]^{\mathrm{T}}=\left[\begin{array}{ll}
x^{\mathrm{T}} & w^{\mathrm{T}}
\end{array}\right]\left[\begin{array}{cc}
\Psi_{11} & \Psi_{12} \\
* & \Psi_{22}
\end{array}\right]\left[\begin{array}{c}
x \\
w
\end{array}\right]<0,
$$

where

$$
\begin{aligned}
\Psi_{11}= & \dot{P}+\left[A+\Delta_{A}+\left(B+\Delta_{B}\right)\left(K+\Delta_{K}\right)\right]^{\mathrm{T}} P \\
& +P\left[A+\Delta_{A}+\left(B+\Delta_{B}\right)\left(K+\Delta_{K}\right)\right]+M \\
& +\left[K+\Delta_{K}\right]^{\mathrm{T}} N\left[K+\Delta_{K}\right]+C^{\mathrm{T}} Q C, \\
\Psi_{12}= & P\left[E+\Delta_{E}\right]-C^{\mathrm{T}} Q D-C^{\mathrm{T}} S, \\
\Psi_{22}= & -D^{\mathrm{T}} Q D-2 D^{\mathrm{T}} S-R .
\end{aligned}
$$

Pre-multiplying and post-multiplying the matrix $\Psi$ with the block $\operatorname{diag}\{X, I\}$, where $X=P^{-1}, Y=K \cdot P^{-1}=K X$. Then the following matrix inequality holds:

$$
\left[\begin{array}{cc}
\Lambda_{11} & \Lambda_{12} \\
* & \Lambda_{22}
\end{array}\right]<0
$$

where

$$
\begin{aligned}
\Lambda_{11}= & X\left[A+\Delta_{A}+\left(B+\Delta_{B}\right)\left(K+\Delta_{K}\right)\right]^{\mathrm{T}} \\
& +\left[A+\Delta_{A}+\left(B+\Delta_{B}\right)\left(K+\Delta_{K}\right)\right] X+X \dot{P} X \\
& +X M X+X\left[K+\Delta_{K}\right]^{\mathrm{T}} N\left[K+\Delta_{K}\right] X+X C^{\mathrm{T}} Q C X, \\
\Lambda_{12}= & {\left[E+\Delta_{E}\right]-X C^{\mathrm{T}} Q D-X C^{\mathrm{T}} S, } \\
\Lambda_{22}= & -D^{\mathrm{T}} Q D-2 D^{\mathrm{T}} S-R .
\end{aligned}
$$

Applying Lemma 2 and Remark 2, we have $X \dot{P} X<0$.

Denote $W=X A^{\mathrm{T}}+A X+Y^{\mathrm{T}} B^{\mathrm{T}}+B Y$.
The sufficient condition for matrix inequality (22) to be held is to change term $\Lambda_{11}$ as follows:

$$
\begin{aligned}
\Lambda_{11}= & X A^{\mathrm{T}}+A X+Y^{\mathrm{T}} B^{\mathrm{T}}+B Y+X\left\{\Delta_{A}+\Delta_{B} K+B \Delta_{K}\right. \\
& \left.+\Delta_{B} \Delta_{K}\right\}^{\mathrm{T}}+\left\{\Delta_{A}+\Delta_{B} K+B \Delta_{K}+\Delta_{B} \Delta_{K}\right\} X \\
& +X M X+Y^{\mathrm{T}} N Y+X \Delta_{K}^{\mathrm{T}} N Y+Y^{\mathrm{T}} N \Delta_{K} X \\
& +X \Delta_{K}^{\mathrm{T}} N \Delta_{K} X+X C^{\mathrm{T}} Q C X \\
= & W+\left\{\Delta_{A}+\Delta_{B} K\right\} X+X\left\{\Delta_{A}+\Delta_{B} K\right\}^{\mathrm{T}} \\
& +X\left\{B \Delta_{K}+\Delta_{B} \Delta_{K}\right\}^{\mathrm{T}}+\left\{B \Delta_{K}+\Delta_{B} \Delta_{K}\right\} X \\
& +\left\{X \Delta_{K}^{\mathrm{T}} N Y+Y^{\mathrm{T}} N \Delta_{K} X\right\}+\left\{X M X+Y^{\mathrm{T}} N Y\right. \\
& \left.+X \Delta_{K}^{\mathrm{T}} N \Delta_{K} X+X C^{\mathrm{T}} Q C X\right\},
\end{aligned}
$$

By applying Lemma 1 to Equation (25) and using Assumption 1 , we obtain

$$
\begin{aligned}
& \left\{\Delta_{A}+\Delta_{B} K\right\} X+X\left\{\Delta_{A}+\Delta_{B} K\right\}^{\mathrm{T}} \\
& =X\left[\begin{array}{ll}
I & K^{\mathrm{T}}
\end{array}\right]\left[\begin{array}{l}
\Delta_{A}^{\mathrm{T}} \\
\Delta_{B}^{\mathrm{T}}
\end{array}\right]+\left[\begin{array}{ll}
\Delta_{A} & \Delta_{B}
\end{array}\right]\left[\begin{array}{c}
I \\
K
\end{array}\right] X \\
& \leq \alpha_{1}\left[\begin{array}{ll}
\Delta_{A} & \Delta_{B}
\end{array}\right]\left[\begin{array}{l}
\Delta_{A}^{\mathrm{T}} \\
\Delta_{B}^{\mathrm{T}}
\end{array}\right]+\alpha_{1}^{-1} X\left[\begin{array}{ll}
I & K^{\mathrm{T}}
\end{array}\right]\left[\begin{array}{l}
I \\
K
\end{array}\right] X \\
& \leq \alpha_{1}\left(\gamma_{A}+\gamma_{B}\right) I+\alpha_{1}^{-1} X\left[\begin{array}{ll}
I & K^{\mathrm{T}}
\end{array}\right]\left[\begin{array}{l}
I \\
K
\end{array}\right] X, \\
& X \Delta_{K}^{\mathrm{T}} B^{\mathrm{T}}+B \Delta_{K} X \leq \alpha_{2} X \Delta_{K}^{\mathrm{T}} \Delta_{K} X \\
& \quad+\alpha_{2}^{-1} B B^{\mathrm{T}} \leq \alpha_{2} \gamma_{K} X^{2}+\alpha_{2}^{-1} B B^{\mathrm{T}}, \\
& X \Delta_{K}^{\mathrm{T}} \Delta_{B}^{\mathrm{T}}+\Delta_{B} \Delta_{K} X \leq \alpha_{3} X \Delta_{K} \Delta_{K}^{\mathrm{T}} X \\
& \quad+\alpha_{3}^{-1} \Delta_{B} \Delta_{B}^{\mathrm{T}} \leq \alpha_{3} \gamma_{K} X^{2}+\alpha_{3}^{-1} \gamma_{B} I, \\
& X \Delta_{K}^{\mathrm{T}} N Y+Y^{\mathrm{T}} N \Delta_{K} X \leq \alpha_{4} X \Delta_{K}^{\mathrm{T}} \Delta_{K} X \\
& \quad+\alpha_{4}^{-1} Y^{\mathrm{T}} N^{2} Y \leq \alpha_{4} \gamma_{K} X^{2}+\alpha_{4}^{-1} Y^{\mathrm{T}} N^{2} Y, \\
& X \Delta_{K}^{\mathrm{T}} N \Delta_{K} X \leq X \Delta_{K}^{\mathrm{T}} \Delta_{K} X \cdot \lambda_{\max }(N) \leq \lambda_{\max }(N) \gamma_{K} X^{2} .
\end{aligned}
$$

Therefore, we have

$$
\begin{aligned}
\Lambda_{11} \leq & W+\alpha_{1}\left(\gamma_{A}+\gamma_{B}\right) I+\alpha_{1}^{-1} X\left[\begin{array}{ll}
I & K^{\mathrm{T}}
\end{array}\right]\left[\begin{array}{c}
I \\
K
\end{array}\right] X \\
& +\alpha_{2} \gamma_{K} X^{2}+\alpha_{2}^{-1} B B^{\mathrm{T}}+\alpha_{3} \gamma_{K} X^{2}+\alpha_{3}^{-1} \gamma_{B} I \\
& +\alpha_{4} \gamma_{K} X^{2}+\alpha_{4}^{-1} Y^{\mathrm{T}} N^{2} Y+\left\{X M X+Y^{\mathrm{T}} N Y\right. \\
& \left.+\lambda_{\max }(N) \gamma_{K} X^{2}+X C^{\mathrm{T}} Q C X\right\}, \\
\Lambda_{12}= & {\left[E+\Delta_{E}\right]-X C^{\mathrm{T}} Q D-X C^{\mathrm{T}} S, } \\
\Lambda_{22}= & -D^{\mathrm{T}} Q D-2 D^{\mathrm{T}} S-R .
\end{aligned}
$$

Using Lemma 1 and Assumption 1, we have

$$
\left[\begin{array}{cc}
0 & \Delta_{E} \\
\Delta_{E}^{\mathrm{T}} & 0
\end{array}\right] \leq\left[\begin{array}{cc}
\alpha_{5} \Delta_{E} \Delta_{E}^{\mathrm{T}} & 0 \\
0 & \alpha_{5}^{-1} I
\end{array}\right] \leq\left[\begin{array}{cc}
\alpha_{5} \gamma_{E} I & 0 \\
0 & \alpha_{5}^{-1} I
\end{array}\right]
$$


Therefore, by applying the results of Equations (24)-(29) to Equation (22), we find:

$$
\left[\begin{array}{cc}
\Theta_{11} & \Theta_{12} \\
* & \Theta_{22}
\end{array}\right]<0,
$$

where

$$
\begin{aligned}
\Theta_{11}= & W+\left[\alpha_{1} \gamma_{A}+\alpha_{1} \gamma_{B}+\alpha_{5} \gamma_{E}+\alpha_{3}^{-1} \gamma_{B}\right] I \\
& +\alpha_{1}^{-1} X\left[\begin{array}{ll}
I & K^{\mathrm{T}}
\end{array}\right]\left[\begin{array}{c}
I \\
K
\end{array}\right] X+\alpha_{4}^{-1} Y^{\mathrm{T}} N^{2} Y \\
& +\left[\alpha_{2}+\alpha_{3}+\alpha_{4}+\lambda_{\max }(N)\right] \gamma_{K} X^{2} \\
& +\alpha_{2}^{-1} B B^{\mathrm{T}}+\left\{X M X+Y^{\mathrm{T}} N Y+X C^{\mathrm{T}} Q C X\right\} \\
= & W+\left[\alpha_{1} \gamma_{A}+\alpha_{1} \gamma_{B}+\alpha_{5} \gamma_{E}+\alpha_{3}^{-1} \gamma_{B}\right] I \\
& +X\left\{\alpha_{1}^{-1} I+\left[\alpha_{2}+\alpha_{3}+\alpha_{4}+\lambda_{\max }(N)\right] \gamma_{K} I\right. \\
& \left.+M+C^{\mathrm{T}} Q C\right\} X \\
& +Y^{\mathrm{T}}\left\{\alpha_{1}^{-1}+\alpha_{4}^{-1} N^{2}+N\right\} Y+\alpha_{2}^{-1} B B^{\mathrm{T}}, \\
\Theta_{12}= & E-X C^{\mathrm{T}} Q D-X C^{\mathrm{T}} S, \\
\Theta_{22}= & -D^{\mathrm{T}} Q D-2 D^{\mathrm{T}} S-R+\alpha_{5}^{-1} I .
\end{aligned}
$$

By applying the Schur complement, we conclude the final LMI solution as

$$
\left[\begin{array}{cccc}
\Upsilon_{11} & \Upsilon_{12} & X & Y^{\mathrm{T}} \\
* & \Upsilon_{22} & 0 & 0 \\
* & * & \Upsilon_{33} & 0 \\
* & * & * & \Upsilon_{44}
\end{array}\right]<0,
$$

where

$$
\begin{aligned}
\Upsilon_{11}= & W+\left[\alpha_{1} \gamma_{A}+\alpha_{1} \gamma_{B}+\alpha_{5} \gamma_{E}+\alpha_{3}^{-1} \gamma_{B}\right] I+\alpha_{2}^{-1} B B^{\mathrm{T}} \\
= & X A^{\mathrm{T}}+A X+Y^{\mathrm{T}} B^{\mathrm{T}}+B Y+\alpha_{2}^{-1} B B^{\mathrm{T}} \\
& +\left[\alpha_{1} \gamma_{A}+\alpha_{1} \gamma_{B}+\alpha_{5} \gamma_{E}+\alpha_{3}^{-1} \gamma_{B}\right] I, \\
\Upsilon_{12}= & E-X C^{\mathrm{T}} Q D-X C^{\mathrm{T}} S, \\
\Upsilon_{22}= & -D^{\mathrm{T}} Q D-2 D^{\mathrm{T}} S-R+\alpha_{5}^{-1} I, \\
\Upsilon_{33}= & -\left\{\alpha_{1}^{-1} I+\left[\alpha_{2}+\alpha_{3}+\alpha_{4}+\lambda_{\max }(N)\right] \gamma_{K} I\right. \\
& \left.+M+C^{\mathrm{T}} Q C\right\}^{-1}, \\
\Upsilon_{44}= & -\left\{\alpha_{1}^{-1}+\alpha_{4}^{-1} N^{2}+N\right\}^{-1} .
\end{aligned}
$$

Since positive constants $\alpha_{1}, \ldots, \alpha_{5}$ are arbitrary, choosing all of them as 1, we obtain Equation (16). Therefore, if LMI (16) holds, the inequality (7) is satisfied. This concludes the proof of the theorem.

Remark 3 At this point, it is to be noted that other choices of positive constants $\alpha_{1}, \ldots, \alpha_{5}$ are possible and can be tried if the value 1 for all these constants does not work.

\section{Application to the inverted pendulum on a cart}

The inverted pendulum on a cart problem (Wang \& Yaz, 2010) is used for testing the novel robust and resilient state-dependent LMI approach to compare the performance. Using the Euler-Lagrange equation technique, the complete equations of motion for the inverted pendulum on a cart can be reached as

$$
\begin{aligned}
& (M+m) \ddot{x}+b \dot{x}+m L \ddot{\theta} \cos (\theta)-m L \dot{\theta} \sin (\theta)=F, \\
& \left(I+m L^{2}\right) \ddot{\theta}+m g L \sin (\theta)+m L \ddot{x} \cos (\theta)=0 .
\end{aligned}
$$

The following system parameters are assumed:

$$
\begin{aligned}
& M=0.5 \mathrm{~kg}, m=0.5 \mathrm{~kg}, b=0.1 \mathrm{~N} \frac{\mathrm{s}}{m}, \\
& L=0.3 \mathrm{~m}, I=0.06 \mathrm{~kg} \mathrm{~m}^{2},
\end{aligned}
$$$$
\text { Sampling time: } T=0.01 \mathrm{~s} \text {. }
$$

Denote the following state variables:

$$
x_{1}=x(t), x_{2}=\dot{x}(t), x_{3}=\theta(t), x_{4}=\dot{\theta}(t) .
$$

The following initial conditions are assumed:

$$
x_{1}=1, x_{2}=0, x_{3}=\pi / 4, x_{4}=0 .
$$

The following design parameters are chosen to satisfy different mixed performance criteria:

Mixed NLQR $-H_{\infty}$ design (predominant NLQR)

$$
\begin{aligned}
C & =\left[\begin{array}{llll}
0.01 & 0.01 & 0.01 & 0.01
\end{array}\right], D=[0.01], M=I_{4}, \\
N & =1, Q=1, S=0, R=-1 .
\end{aligned}
$$

Mixed NLQR $-H_{\infty}$ design (predominant $H_{\infty}$ )

$C=\left[\begin{array}{llll}1 & 1 & 1 & 1\end{array}\right], D=[1], M=0.01 \times I_{4}, N=0.01$, $Q=1, S=0, R=-10$.

Mixed NLQR $-H_{\infty}-$ positive real design (NLQR passivity)

$$
\begin{aligned}
& C=\left[\begin{array}{llll}
1 & 1 & 1 & 1
\end{array}\right], D=[1], M=I_{4}, N=1, \\
& Q=0.01, S=1, R=0.01 .
\end{aligned}
$$

All of the above mixed criteria control performance results are shown in Figures 1-5, in comparison with the traditional LQR technique based on linearization. From these figures, we find that the novel state-dependent LMI control has better performance compared with the traditional LQR technique based on linearization. Especially, Figures 3 and 4 show that the traditional $\mathrm{LQR}$ technique loses control of the angle and angular velocities of the pendulum, respectively. Figure 5 shows that the highest magnitude of control is needed by the predominant $H_{\infty}$ control and the lowest control magnitude is needed by the linearization-based LQR technique. 


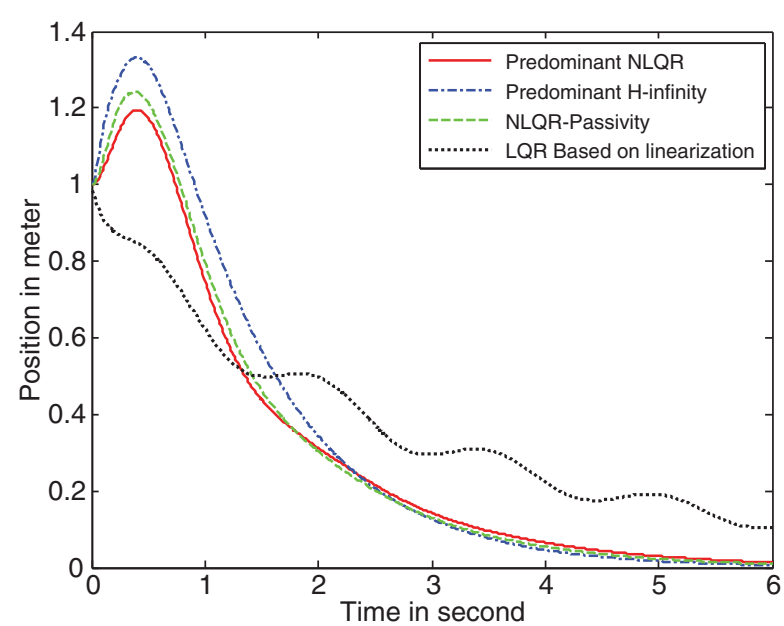

Figure 1. Position trajectory of the inverted pendulum.

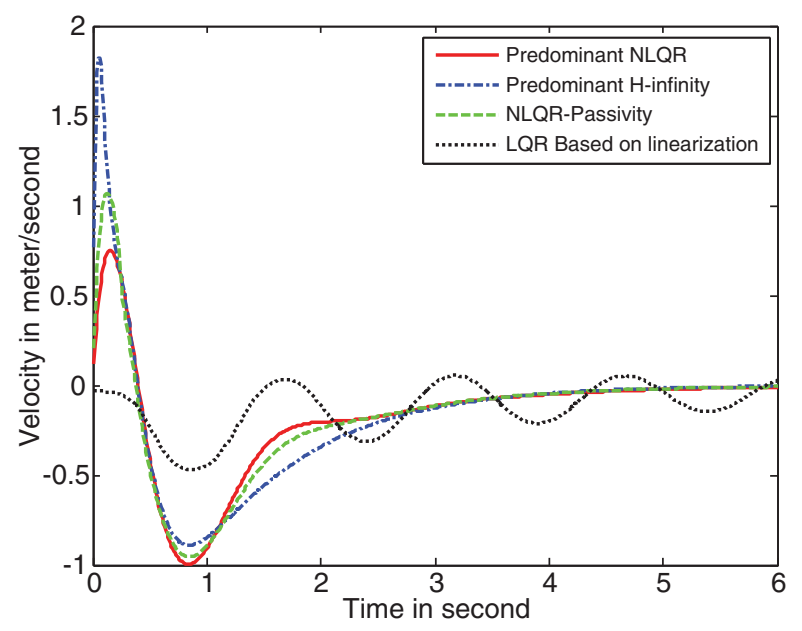

Figure 2. Velocity trajectory of the inverted pendulum.

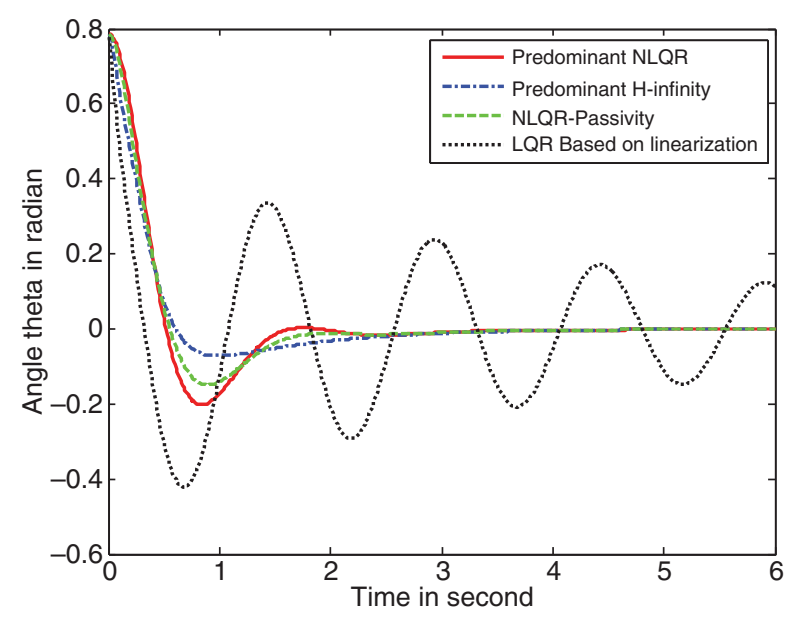

Figure 3. Angle "theta" trajectory of the inverted pendulum.

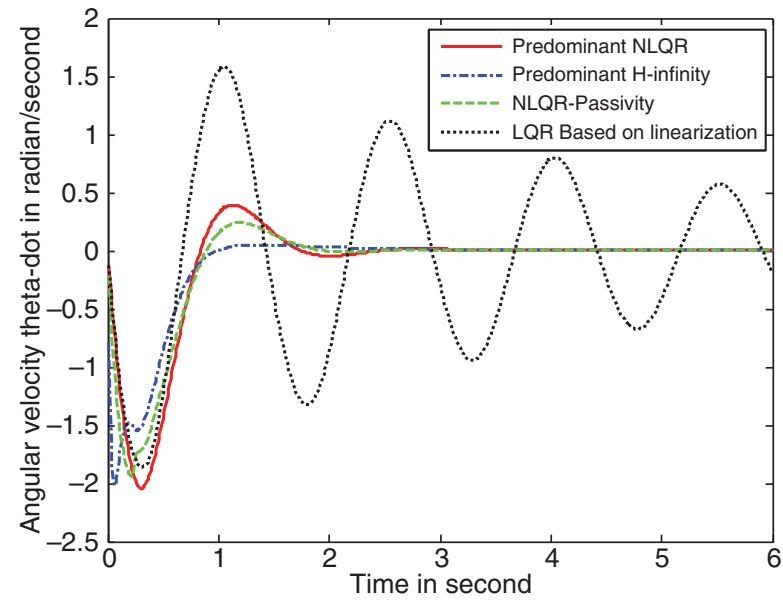

Figure 4. Angular velocity trajectory of the inverted pendulum.

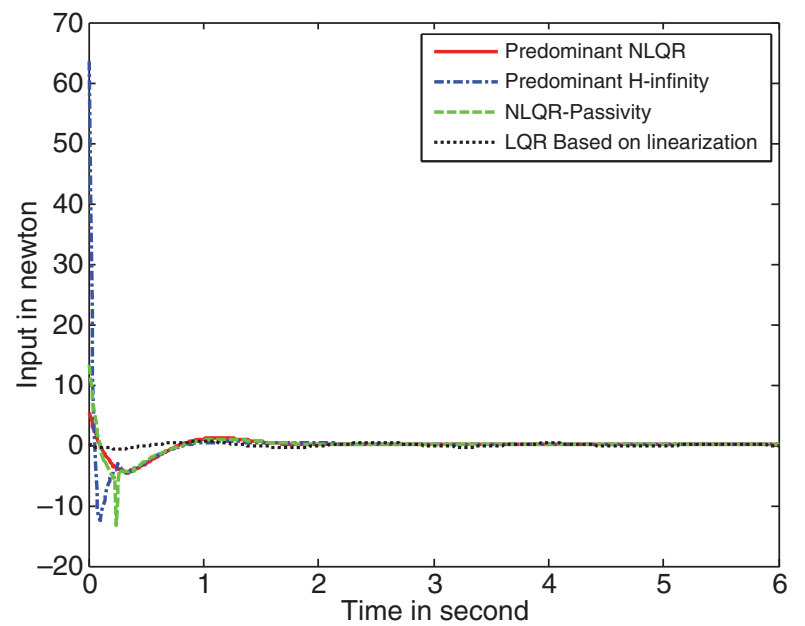

Figure 5. Control input.

\section{Conclusions}

This paper addresses nonlinear system control design with general NLQR and quadratic dissipative criteria to achieve asymptotic stability, quadratic optimality and strict quadratic dissipativeness. For systems with unstructured but bounded uncertainty, the LMI-based sufficient conditions are derived for the solution of general performance criteria control. Our results unify the existing results on SDRE control, robust $H_{\infty}$, positive real control and sector-bounded control. The relative weighting matrices of these criteria can be achieved by choosing different weighing coefficient matrices. The optimal control can be obtained by solving LMI at each time step. The inverted pendulum on a cart control, which is a benchmark under-actuated nonlinear control system, is used as an example to demonstrate its effectiveness and robustness of the proposed method. The simulation studies show that the proposed method provides a satisfactory alternative to the existing nonlinear control approaches. 


\section{Acknowledgements}

We thank the reviewers for their remarks, which have improved the quality of the paper. This research has been financially supported by a grant from NITC (National Institute for Transportation and Communities), which is gratefully acknowledged.

\section{References}

Basar, T., \& Bernhard, P. (1995). H-infinity optimal control and related minimax design problems $-A$ dynamic game approach (2nd ed.). Boston: Birkhauser.

Boyd, S., Ghaoui, L. E., Feron, E., \& Balakrishnan, V. (1994). Linear matrix inequalities in system and control theory, SIAM Studies in Applied Mathematics. Philadelphia, PA: SIAM.

Cloutier, J. R. (1997). State-dependent Riccati equation techniques: An overview. Proceedings of the 1997 American Control Conference, pp. 932-936, Albuquerque, MN.

Cloutier, J. R., D’Souza, C. N., \& Mracek, C. P. (1996). Nonlinear regulation and nonlinear $H$-infinity control via the statedependent Riccati equation technique: Part 1 theory, part 2 examples. Proceedings of 1 st international conference on nonlinear problems in aviation and aerospace, pp. 117-141, Daytona Beach, FL.

Doyle, J. C., Glover, K., Khargonekar, P. P., \& Francis, B. A. (1989). State space solution to the standard $\mathrm{H} 2$ and $\mathrm{H}$-infinity control problems. IEEE Transactions on Automatic Control, $34,831-847$

Gupta, S., \& Joshi, S. M. (1994). Some properties and stability results for sector bounded LTI systems. Proceedings of 33rd IEEE conference on decision and control, pp. 2973-2978, Orlando, USA

Haddad, W. M., \& Bernstein, D. S. (1991). Robust stabilization with positive real uncertainty, beyond the small gain theorem. Systems and Control Letters, 17, 191-208.

Hill, D. J., \& Moylan, P. J. (1975). Cyclo-dissipativeness, dissipativeness and losslessness for nonlinear dynamical systems (Technical Report EE7526). Department of Electrical and Computer Engineering, University of Newcastle, Australia.

Hill, D. J., \& Moylan, P. J. (1976). Stability of nonlinear dissipative systems. IEEE Transactions on Automatic Control, 21, 708-711.

Hill, D. J., \& Moylan, P. J. (1980). Dissipative dynamical systems: Basic input-output and state properties. Journal of the Franklin Institute, 309, 327-357.

Huang, Y., \& Lu, W-M. (1996). Nonlinear optimal control: alternatives to Hamilton-Jacobi equation. Proceedings of 35th conference on decision and control, pp. 3942-3947, Kobe, Japan.

Khargonekar, P. P., Petersen, I. R., \& Zhou, K. (1990). Robust stabilization of uncertain linear systems: quadratic stabilizability and $\mathrm{H}$-infinity control theory. IEEE Transactions on Automatic Control, 35, 356-361.
Mohseni, J., Yaz, E., \& Olejniczak, K. (1998). State dependent LMI control of discrete-time nonlinear systems. Proceedings of the 37th IEEE conference on decision and control, pp. 4626-4627, Tampa, FL.

Petersen, I. R. (1987). A stabilization algorithm for a class of uncertain systems. Systems and Control Letters, 8, 181-188.

Safonov, M. G., Jonckheere, E. A., Verma, M., \& Limebeer, D. J. N. (1987). Synthesis of positive real multivariable feedback systems. International Journal of Control, 45, 817-842.

Shim, D. (1996). Equivalence between positive real and normbounded uncertainty. IEEE Transactions on Automatic Control, 41, 1190-1193.

Sun, W., Khargonekar, P. P., \& Shim, D. (1994). Solution to the positive real control problem for linear time-invariant systems. IEEE Transactions on Automatic Control, 39, 2034-2046.

Tan, Z., Soh, Y. C., \& Xie, L. (2000). Dissipative control of linear discrete-time systems with dissipative uncertainty. International Journal of Control, 73(4), 317-328.

Van der Shaft, A. J. (1993). Nonlinear state space H-infinity control theory. In H. J. Trentelman \& J. C. Willems (Eds.), Perspectives in control, pp. 153-190. Groningen: Birkhauser.

Wang, X., \& Yaz, E. E. (2009). The state dependent control of continuous time nonlinear systems with mixed performance criteria. Proceedings of IASTED international conference on identification and control applications, pp. 98-102, Honolulu, HI.

Wang, X., Yaz, E. E., \& Jeong, C. S. (2010). Robust nonlinear feedback control of discrete-time systems with mixed performance criteria. Proceedings of American control conference, pp. 6357-6362, Baltimore, MD.

Wang, X., Yaz, E. E., \& Yaz, Y. I. (2010). Robust and resilient state dependent control of continuous time nonlinear systems with general performance criteria. Proceedings of the 49th IEEE conference on decision and control, pp. 603-608, Atlanta, GA

Wang, X., Yaz, E. E., \& Yaz, Y. I. (2011). Robust and resilient state dependent control of discrete time nonlinear systems with general performance criteria. Proceedings of the 18th IFAC congress, pp. 10904-10909, Milano, Italy.

Willems, J. C. (1972a). Dissipative dynamical systems - Part 1: General theory. Archives for Rational Mechanics and Analysis, 45, 321-351.

Willems, J. C. (1972b). Dissipative dynamical systems - Part 2: Linear systems with quadratic supply rates'. Archives for Rational Mechanics and Analysis, 45, 352-393.

Xie, S., Xie, L., \& De Souza, C. E. (1998). Robust dissipative control for linear systems with dissipative uncertainty. International Journal of Control, 70(2), 169-191.

Zhou, K., \& Khargonekar, P. P. (1988). An algebraic Riccati equation approach to H-infinity optimization. Systems and Control Letters, 11, 85-91. 